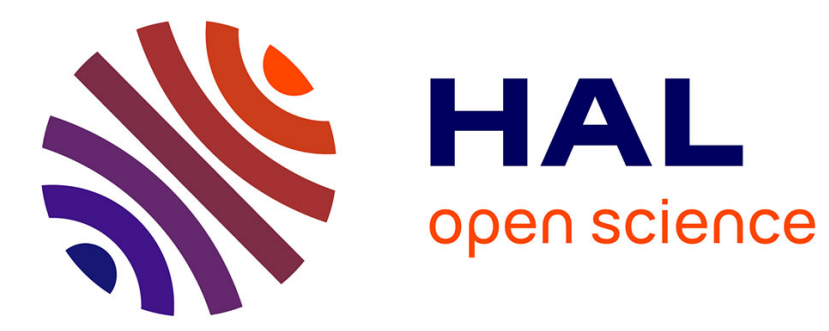

\title{
Expérimentation de capteurs solaires à air à absorbeur poreux
}

\author{
E. Rauzier
}

\section{To cite this version:}

E. Rauzier. Expérimentation de capteurs solaires à air à absorbeur poreux. Revue de Physique Appliquée, 1980, 15 (3), pp.411-415. 10.1051/rphysap:01980001503041100 . jpa-00244745

\section{HAL Id: jpa-00244745 https://hal.science/jpa-00244745}

Submitted on 1 Jan 1980

HAL is a multi-disciplinary open access archive for the deposit and dissemination of scientific research documents, whether they are published or not. The documents may come from teaching and research institutions in France or abroad, or from public or private research centers.
L'archive ouverte pluridisciplinaire HAL, est destinée au dépôt et à la diffusion de documents scientifiques de niveau recherche, publiés ou non, émanant des établissements d'enseignement et de recherche français ou étrangers, des laboratoires publics ou privés. 


\title{
Expérimentation de capteurs solaires à air à absorbeur poreux
}

\author{
E. Rauzier \\ C.N.R.S., Laboratoire d'Ecothermique Solaire, Observatoire de Nice, France \\ (Reçu le 28 septembre 1979, révisé le 23 novembre 1979, accepté le 26 novembre 1979)
}

\begin{abstract}
Résumé. - Afin de diminuer les pertes par rayonnement des capteurs solaires à air classiques, nous avons conçu et expérimenté différents capteurs à absorbeur poreux. Pour les meilleurs matériaux (tôle déployée à maille écrasée, verre sélectif pilé et mousse plastique réticulée) les rendements sont de $15 \%$ plus élevés que pour les capteurs traditionnels.
\end{abstract}

\begin{abstract}
In order to lower the radiative losses of a traditional solar air collector, different porous absorber collectors were designed and experimented. The best materials used seems to be flat meshes expanded metal sheets, crushed selective glass and reticulate plastic foam : the efficiencies are $15 \%$ higher than those of traditional air collectors.
\end{abstract}

1. Introduction. - Le captage de l'énergie solaire est d'autant plus efficace que la température de l'absorbeur est plus basse. Cette remarque simple a conduit le Groupe Ecothermique Solaire de Nice à étudier des capteurs à basse température et, des systèmes de stockage thermique à chaleur latente [1].

Cette démarche conduit à privilégier les systèmes de captages à gain direct, l'emploi de serres accolées à l'habitat et les capteurs à air. L'emploi de ces capteurs permet de réconcilier pour un faible coût les nécessités d'adaptations architecturales et les nécessités thermiques de rendement de faible inertie et d'absence d'effet de seuil.

2. Les capteurs à absorbeur poreux. - Le capteur à air le plus élémentaire est constitué d'un absorbeur de tôle noire au contact duquel on fait circuler de l'air à l'abri d'un vitrage. Le rendement d'un tel capteur est limité à $50 \%$ dans les conditions les plus favorables [2] : $1 \mathrm{kWh} / \mathrm{m}^{2}, \Delta T=55^{\circ} \mathrm{C}$.

Ce rendement peut être amélioré par l'emploi de revêtements sélectifs, par une modification de la géométrie du capteur [3] et notamment par une circulation du flux d'air perpendiculaire au rayonnement (absorbeur poreux).

De tels absorbeurs par l'isolation dynamique (Cadiergues [4]) qu'ils créent sur leur face avant, limitent de façon très efficace les déperditions et atteignent à un moindre coût des rendements comparables à ceux des structures antirayonnantes et anticonvectives de Francia [5].

Dans leurs travaux sur des capteurs poreux à struc- ture de nids d'abeille, Lalude, Buckberg et Edwards [6], [7] annoncent par exemple des rendements de $63 \%$ pour les mêmes conditions favorables que ci-dessus.

D'autres types de capteurs poreux existent, citons notamment :

- Les capteurs à absorbeurs étagés en verre de Selcuk [8].

- Les capteurs à grilles de métal déployé étudiés par Chiou, El Wakil et Duffie [9].

- Les murs claustras de Pince [10] qui donnent une adaptation architecturale intéressante de ces systèmes.

- La Société Bertin [11] a également étudié l'adaptation à un immeuble de capteurs poreux.

L'ensemble des systèmes à capteurs poreux a été étudié théoriquement par Hamid et Beckman [12, $13,14]$.

Notre but est de concevoir et d'expérimenter un élément de mur actif assurant efficacement, la capture, le stockage et la restitution de l'énergie solaire tout en y associant les fonctions d'isolation et de renouvellement d'air.

La présente étude décrit les expérimentations préliminaires effectuées pour le choix du matériau adapté à la confection d'un absorbeur poreux. Nous appuyons les résultats expérimentaux à l'aide d'une modélisation simple.

3. Description du dispositif expérimental. - 3.1 PrinCIPES GÉNÉRAUX. - Afin de rendre l'expérimen- 
tation aussi reproductible que possible le dispositif fonctionne avec un éclairage artificiel et toutes les mesures sont prises par référence à un même étalonnage.

Dans ce genre d'expérimentation la précision absolue est assez faible. Nous nous sommes donc efforcés de travailler le plus comparativement possible.

De plus, le banc d'essai a été placé dans une salle isotherme (grande inertie thermique, murs de $4 \mathrm{~m}$ d'épaisseur, température d'air homogénéisée par ventilation). Enfin pour éviter les effets parasites dus à l'échauffement exagéré du vitrage par absorption d'un rayonnement infrarouge plus intense que dans le spectre solaire, de nombreuses précautions ont été prises pour éliminer ce rayonnement : emploi de filtres réfléchissants dans l'infrarouge, ventilation violente au niveau du filtre et du vitrage.

3.2 Disposition géométriQue. - Pour la commodité des essais le banc d'expérimentation est horizontal, ce qui est légitime du fait de l'exploitation comparative des résultats.

L'absorbeur a une surface de $0,2 \mathrm{~m}^{2}$. Il est monté dans un cadre isolant amovible. Les absorptions parasites sont très fortement réduites par des diaphragmes de polyéthylène aluminisé (Rexotherm). I'ne modification mineure permet de passer d'un absorbeur poreux à un capteur classique.

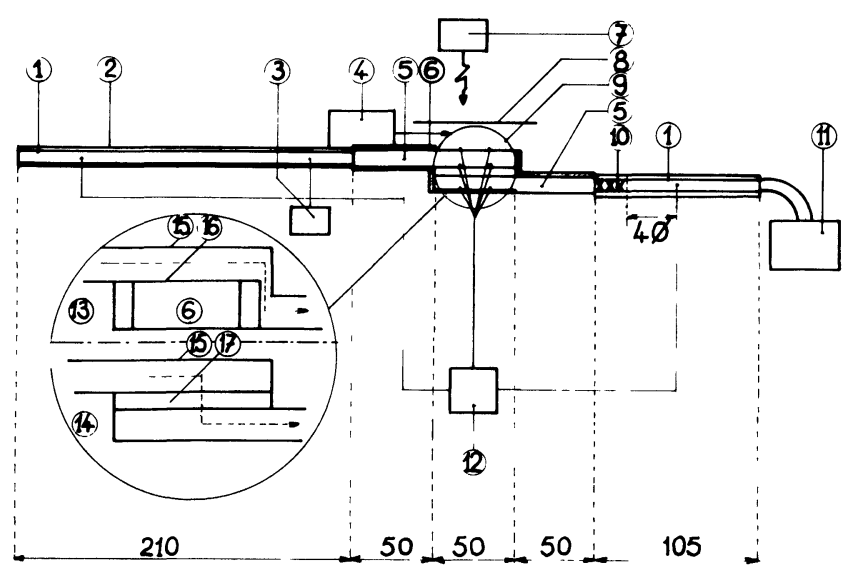

Fig. 1. - Coupe longitudinale du banc d'essai de capteurs à air. Echelle : $5 \mathrm{~cm}$ pour $3 \mathrm{~m}$. 1 : Conduites PVC d'amenée et d'évacuation de l'air. 2 : Isolation de laine de verre. 3 : Dispositif de mesure des vitesses. 4 : Ventilation. 5 : Cônes de raccordement 6 : Isolation de polystyrène. 7 : Eclairage artificiel. 8 : Diaphragme et filtre infrarouge. 9 : Ensemble capteur et ses thermistances. 10 : Mélangeur d'air. 11 : Système d'aspiration. 12 : Enregistrement des températures. 13 : Schéma d'un capteur traditionnel 14 : Schéma d'un capteur poreux. 15 : Vitre. 16 : Tôle plate. 17 : Matériau poreux logé dans un tiroir.

[Longitudinal section of the air collectors experimental device. Scale : $5 \mathrm{~cm}$ for $3 \mathrm{~m} .1$ : Inlet and output PVC air-main. 2 : Glasswool insulation. 3 : Speed measure device. 4 : Ventilation. 5 : Junction cone. 6 : Polytirene insulation. 7 : Artificial lightning. 8 : Diaphragm and infrared filter. 9 : Collector and its thermistances. 10 : Air-mixer. 11 : Aspiration. 12 : Temperature recorder. 13 : Traditional collector schema. 14 : Porous collector schema. 15 : Glass cover. 16 : Metal plate. 17 : Porous material set in a drawer.]
La distance vitre absorbeur est de $0,1 \mathrm{~m}$. Les raccordements entre le capteur et les tubulures PVC d'alimentation de $\varnothing 0,08 \mathrm{~m}$ sont faits par des cônes d'ouverture $30^{\circ}$.

L'ensemble du dispositif est décrit en figure 1.

3.3 EClairage ARTIFICIEL. - Il est effectué par 4 lampes Sylvania [15] présentant les caractéristiques ci-dessous :

- lampe tungstène hologène $500 \mathrm{~W}-220 \mathrm{~V}$,

- durée de vie $2000 \mathrm{~h}$,

- température de couleur $3000 \mathrm{~K}$,

- énergie lumineuse émise $W$ liée à la tension $U$ par une relation [15]

$$
W=\alpha U^{1,55} .
$$

Ces lampes sont disposées dans des projecteurs qui fournissent sur un plan horizontal des caractéristiques d'éclairement de coupe trapézoïdale. Elles ont été associées à des distances correspondant aux largeurs à mi-hauteur de ces caractéristiques. Il en résulte un éclairement uniforme à $\pm 10 \%$, ce que nous avons vérifié en faisant une carte des flux avec un pyranomètre. La tension appliquée aux lampes est relevée en permanence et utilisée pour corriger les variations éventuelles de flux reçus.

Après filtrage l'énergie résiduelle moyenne au niveau de l'absorbeur est de $200 \mathrm{~W} / \mathrm{m}^{2} \pm 10 \mathrm{~W} / \mathrm{m}^{2}$. La température extérieure de la vitre est pratiquement constante à $1^{\circ}$ près.

3.4 MeSURE DES TEMPÉRATURES. - On enregistre en permanence sur un MECI les tensions aux bornes de 12 sondes placées dans les canaux d'entrée (1) et de sortie (3) sur les faces interne et externe de la vitre, dans le matériau poreux ( 2 ou 4 ) et éventuellement au fond du capteur.

Ces sondes étalonnées dans un bain d'huile thermostaté sont de type thermistance CTN de résistance $150 \Omega$ à $25^{\circ}$, la variation de résistance est de 5 à $10 \Omega$ par degré dans le domaine d'expérimentation.

Les imprécisions de mesure proviennent essentiellement d'une légère dérive (compensée par des étalonnages répétés) du MECI et des difficultés physiques de définition des températures de surface. Les mesures de température d'entrée et de sortie sont effectuées selon la procédure décrite dans le Manuel de l'ingénieur [16] : sondes placées dans des tuyaux réfléchissants à 4 diamètres d'un mélangeur.

3.5 MeSure deS viTESSES. - La méthode employée est une méthode de fil chaud. On mesure à l'aide d'un pont de Wheatsone l'échauffement d'une thermistance soumise à une tension stabilisée et placée au centre de la buse dans laquelle on veut mesurer le débit.

L'étalonnage de ces températures est effectué pour des vitesses de référence variant de 0,15 à $4 \mathrm{~m} / \mathrm{s}$. Ces vitesses stables sont obtenues en plaçant la sonde sur une platine tournante. Ayant la vitesse au centre de 
la veine de fluide à plus de 10 diamètres de l'entrée, on extrapole le profil des vitesses :

- par un profil parabolique en régime laminaire,

- par un profil de Coantic [17] pour le régime turbulent,

- pour l'écoulement critique nous nous sommes contentés d'une interpolation.

3.6 CONDUITE DES EXPÉRIMENTATIONS. - On a procédé aux mesures point par point pour chaque matériau et pour différentes valeurs du débit ( 8 valeurs de débit par matériau).

Chaque mesure n'est prise en compte qu'après stabilisation complète des températures enregistrées. La mesure complète d'un absorbeur poreux nécessite une dizaine d'heures.

3.7 MATÉRIAUX POREUX ÉTUdiés. - Nous avons recherché des éléments simples et peu coûteux : les paramètres dont nous avons examiné l'influence sont la taille des pores, la transparence, la sélectivité et la conductivité.

Les absorbeurs poreux qui ont été expérimenté peuvent être classés en trois grandes catégories :

- Bons conducteurs : tôles déployées et tôles silo, grillages copeaux de métal.

- Isolants : mousses à pores ouvertes de polyether et polyuréthane (Retipren, billes de polystyrène).

La régularité des dimensions des pores des mousses polyuréthane réticulées et leurs propriétés physiques en font un élément intéressant.

- Matériaux transparents : éclats de verre, sélectifs ou non, de dimensions calibrées.

Tous les échantillons étudiés, à l'exception des verres, ont été peints préalablement par un aérosol de peinture noire mate au polyuréthane.

4. Résultats expérimentaux. - Les courbes des figures 2,3 et 4 donnent une comparaison des performances des capteurs poreux et traditionnel et la comparaison des matériaux poreux. Nous appellons capteur traditionnel une simple tôle non poreuse avec passage d'air entre la tôle et la vitre comme schématisé en figure 1.

\subsection{Performance Relative Des CaPteurs tradi-} TIONNELS ET DES CAPTEURS POREUX. - La figure 2 montre un matériau poreux qui améliore l'efficacité par une diminution des écarts entre la température de l'absorbeur et la température d'air. Les mesures données pour la température de l'absorbeur ne sont pas très réalistes mais prouvent qu'il est meilleur.

Les courbes de la figure 2 montrent une efficacité du capteur poreux qui peut atteindre $75 \%$ à $2 \mathrm{~cm}^{-1}$, valeur très supérieure à l'efficacité du capteur classique, toutes choses égales par ailleurs. Ce rendement opti-

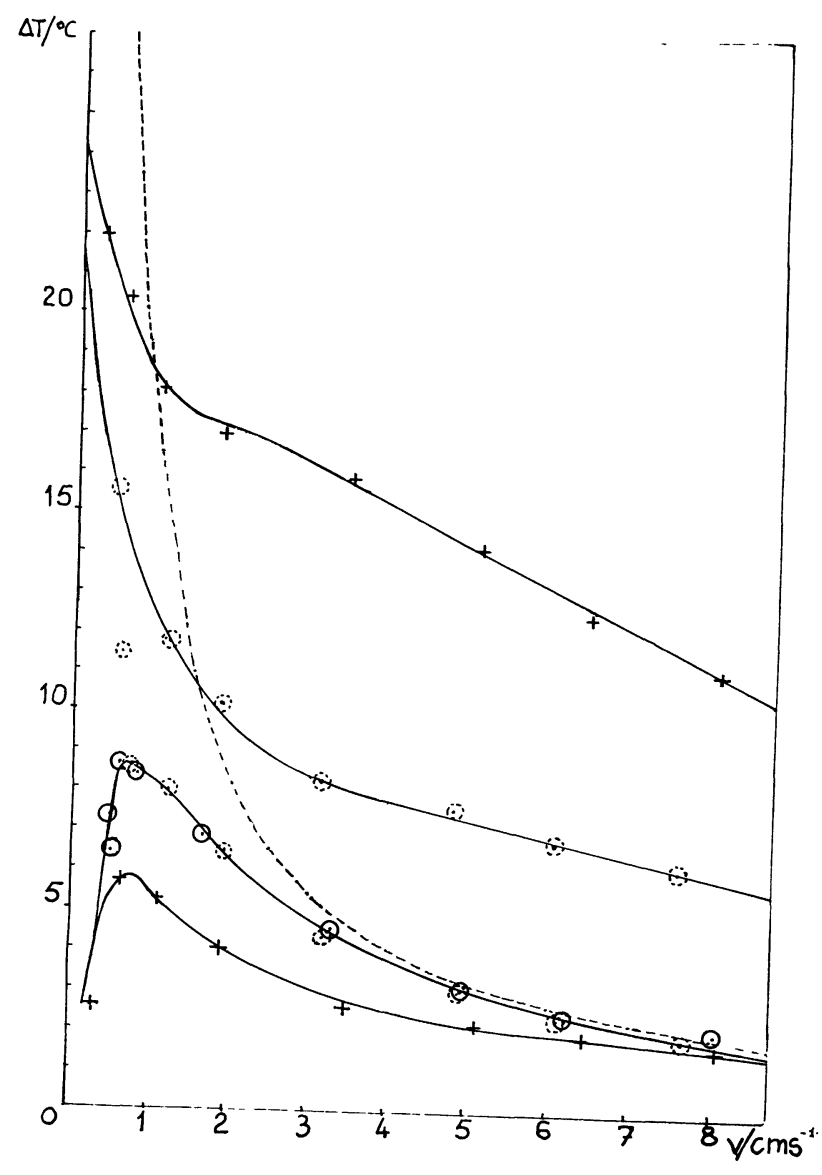

Fig. 2. - Comparaison de deux capteurs à air. +-+-+ Capteur traditionnel. $\mathrm{O}-\mathrm{O}-\mathrm{O}$ Capteur à absorbeur poreux. (Ces ronds en pointillés sont des points de mesure d'une autre expérimentation réalisée avec une sonde de vitesse différente.) ------ Hyperbole $\Delta T=Q_{\text {ray }} / \rho C_{\mathrm{p}} v$ fixant la barre des $100 \%$ de rendement, qui serait atteinte par un capteur sans perte. Pour chaque capteur, la courbe la plus basse représente l'élévation de température de l'air, la courbe la plus haute l'élévation de température du matériau poreux.

[Comparison of two air-collectors. +-+-+ Traditional collector. $\mathrm{O}-\mathrm{O} \longrightarrow$ Porous absorber collector (Retipren foam). (The slippled circles are measure points of another experimentation made with a different speed probe.) ------ Hyperbola $\Delta T=Q_{\text {ray }} / \rho C_{\mathrm{p}} v$ representing a $100 \%$ efficient ideal collector. For each collector, the lower curve is for air temperature rise, the higher one is for absorber temperature rise.]

mum est atteint pour des vitesses d'air relativement faibles qui correspondent encore à une élévation de température suffisante pour des applications à l'habitat.

A plus forte vitesse d'extraction après avoir atteint son maximum, le rendement diminue légèrement par suite de pertes accrues du fait de phénomènes convectifs turbulents.

\subsection{COMParaison DE Divers MATÉriaUX POREUX.} - Le matériau poreux présenté sur les figures 2 et 3 est une mousse polyuréthane réticulée (Retipren) qui a d'excellentes performances. Nous avons comparé plusieurs autres types de matériaux poreux, notam- 
ment des verres pilés (Fig. $3 a$ ) et des tôles déployées (Fig. 3b).

Nous nous sommes efforcés d'appuyer ces résultats à l'aide d'un modèle à deux faisceaux (montant et

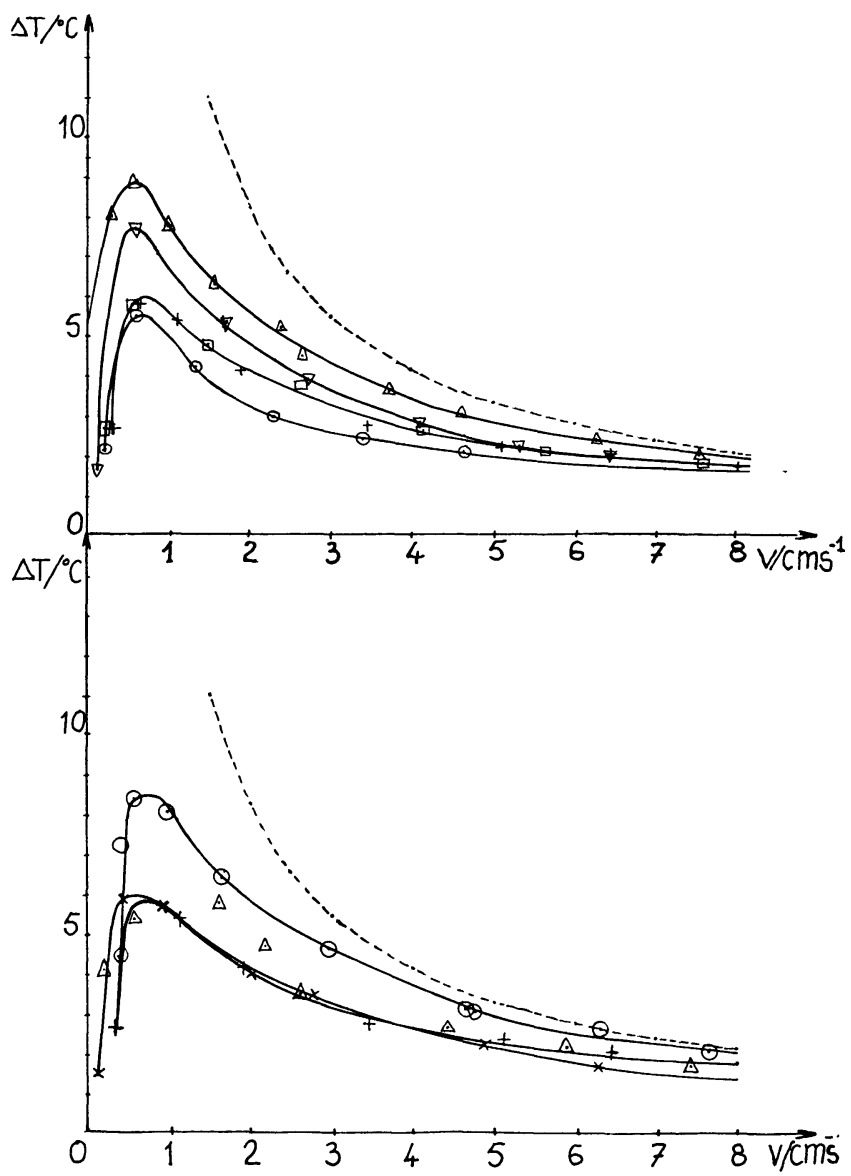

Fig. $3 a$. - Elévation de température d'air pour les capteurs à éclats de verre. +-+-+ Capteur traditionnel. ------ Hyperbole $\Delta T=Q_{\text {ray }} / \rho C_{\mathrm{p}} v$ fixant la barre des $100 \%$ de rendement, qui serait atteinte par un capteur sans perte. $\Delta-\Delta-\Delta$ Eclats de verre sélectif $<1 \mathrm{~cm}^{2}$ et $>3 \mathrm{~mm}^{2} . \nabla-\nabla-\nabla$ Eclats de verre bouteille $<1 \mathrm{~cm}^{2}$ et $>3 \mathrm{~mm}^{2}$. $\square-\square-\square$ Eclats de verre bouteille $>1 \mathrm{~cm}^{2}$. ○-O-O Eclats de verre bouteille $<3 \mathrm{~mm}^{2}$.

[Air temperature rise of a crushed glass collector. +-+-+ Traditional collector. --.---.--.- Hyperbola $\Delta T=Q_{\text {ray }} / \rho C_{\mathrm{p}} v$ representing a $100 \%$ efficient ideal collector. $\Delta-\Delta-\Delta$ Broken selective glass $<1 \mathrm{~cm}^{2}$ and $>3 \mathrm{~mm}^{2} . \nabla-\nabla-\nabla$ Broken bottle glass $<1 \mathrm{~cm}^{2}$ and $>3 \mathrm{~mm}^{2}$. $\square-\square-\square$ Broken bottle glass $>1 \mathrm{~cm}^{2}$. ○一—— Broken bottle glass $<3 \mathrm{~mm}^{2}$.]

Fig. 3b. - Elévation de température d'air par les capteurs à tôles déployées et matériaux conducteurs. +-+-+ Capteur traditionnel. -------- Hyperbole $\Delta T=Q_{\text {ray }} / \rho C_{\mathrm{p}} v$ fixant la barre des $100 \%$ de rendement, qui serait atteinte par un capteur sans perte. ○- - - Tôle déployée à mailles plates et assez fermées (référence : tôle mistral). $\times-\times-\times$ Tôle déployée à grosses mailles très ouvertes (référence : tôle 28-15-15). $\Delta-\Delta-\triangle$ Copeaux de métal.

[Air temperature rise of a collector with expanded metal sheet and conductive materials. +-+-+ Traditional collector. ----- Hyperbola $\Delta T=Q_{\text {ray }} / \rho C_{\mathrm{p}} v$ representing a $100 \%$ efficient ideal collector. $\mathrm{O}-\mathrm{O}-\mathrm{O}$ Expanded metal sheet with flat and rather closed meshes and rather closed (reference : sheet mistral). $x-x-x$ Expanded sheet with large and very opened (reference : sheet 28-15-15). $\triangle-\triangle-\triangle$ Metal cuttings.] descendant) où le matériau est divisé en $n$ couches qui échangent avec les $n-1$ autres du rayonnement par transmission et réflection visible, du rayonnement infrarouge, et échangent avec les 2 voisines de la chaleur par conduction et par déplacement d'air chaud.

Ce modèle est simple et ne prend pas en compte la convection naturelle qui a lieu entre l'absorbeur et la vitre pour de faibles vitesses. Une étude plus poussée est en cours. Toutefois pour $v>1 \mathrm{~cm}^{-1} \mathrm{~s}$, les courbes peuvent être considérées comme valables et surtout elles donnent une idée sur l'influence des différents paramètres.

La figure 4 présente les courbes théoriques pour

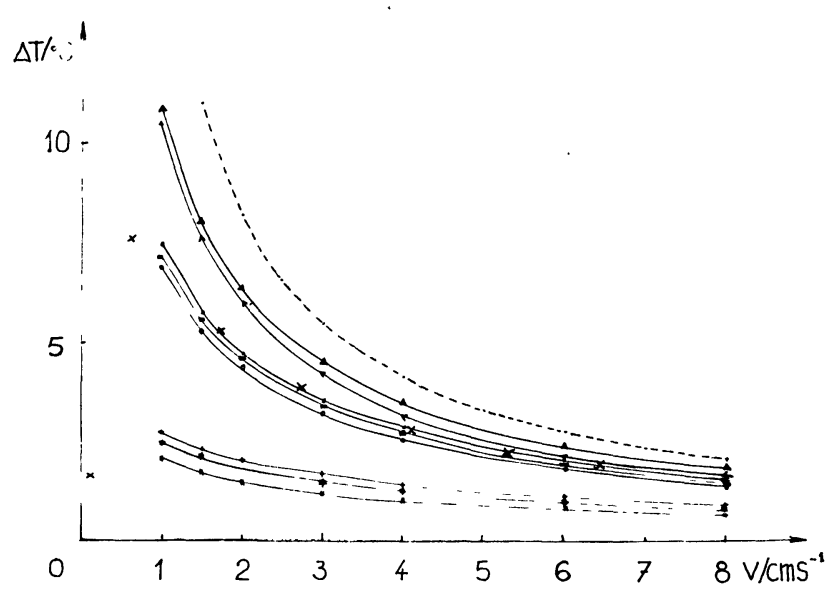

Fig. 4. - Elévation de température de l'air dans le capteur en fonction de la vitesse pour différentes porosités et coefficients d'estimation de l'absorbeur. Dans l'ordre de bas en haut :

$$
\begin{aligned}
& |||||| p=0,9, \quad \beta=0 \mathrm{~m}^{-1} \\
& \text { *-*—* } \quad p=0,9, \quad \beta=100 \mathrm{~m}^{-1} \\
& +-+-+p=0,9, \quad \beta=10000 \mathrm{~m}^{-1} \\
& \text { ○一— } p=0,53, \quad \beta=10000 \\
& \text { - - } p=0,53, \quad \beta=0 \\
& \longrightarrow p=0,53, \quad \beta=100 \\
& <-<-<p=0,1, \quad \beta=30 \\
& \Delta-\Delta-\Delta p=0,1, \quad \beta>100 \text {. }
\end{aligned}
$$

$\times \times \times$ Points de mesure pour le verre pilé $(p=0,53$; $\beta=40 \mathrm{~m}^{-1}$ ) calibré entre $3 \mathrm{mn}^{2}$ et $1 \mathrm{~cm}^{2}$. ----- Hyperbole $\Delta T=Q_{\text {ray }} / \rho C_{\mathrm{p}} v$ fixant la barre des $100 \%$ de rendement, qui serait atteinte par un capteur sans perte.

[Air temperature rise in the collector' versus speed for different porosities and extinction coefficients of the absorber. From bottom to top.

$$
\begin{aligned}
& |1|-|| p=0.9, \quad \beta=0 \mathrm{~m}^{-1} \\
& \text { *—*—* } \quad p=0.9, \quad \beta=100 \mathrm{~m}^{-1} \\
& +-+-+p=0.9, \quad \beta=10000 \mathrm{~m}^{-1} \\
& \text { ○○一 } p=0.53, \beta=10000 \\
& \text { - - - } p=0.53, \beta=0 \\
& \longrightarrow p=0.53, \quad \beta=100 \\
& <-<-<p=0.1, \quad \beta=30 \\
& \Delta-\Delta-\Delta p=0.1, \quad \beta>100 \text {. }
\end{aligned}
$$

$\times \times \times$ Experimental results for crushed glass $(p=0,53$, $\beta=40 \mathrm{~m}^{-1}$ ) pieces surface between $3 \mathrm{mn}^{2}$ and $1 \mathrm{~cm}^{2}$. .-.- Hyperbola $\Delta T=Q_{\text {ray }} / \rho C_{\mathrm{p}} v$ representing a $100 \%$ efficient ideal collector.] 
différentes porosités et différents coefficients d'extinction du rayonnement solaire dans le matériau. Tous les autres paramètres (coefficients de réflexion, taille moyenne des morceaux...) sont ceux de l'expérience avec des éclats de verre de bouteille compris entre $3 \mathrm{~mm}^{2}$ et $1 \mathrm{~cm}^{2}$ dont nous avons porté les points de mesure. A titre d'indication pour ces éclats, on a mesuré $p=0,53$ et $\beta=40 \mathrm{~m}^{-1}$.

Toutes choses égales par ailleurs, la variation des paramètres les plus significatifs fait ressortir que le rendement du capteur est favorisé par :

- une porosité faible,

- un coefficient d'émissivité infrarouge et de réflexion sur les faces tournées vers le soleil les plus faibles possibles,

- une surface d'échange air-matériau importante.

Enfin dans des proportions moins grandes :

- une conductibilité faible,

- une taille moyenne de morceau faible dans le sens vertical,

- un absorbeur épais,

- un coefficient d'absorption important dans le fond du capteur,

- un coefficient d'extinction optimum dépendant de la porosité $\beta=100$ pour $p=0,53$,

- les copeaux de métal et le grillage à grosses mailles sont bien trop poreux pour piéger le rayonnement du fond du capteur et n'offrent pas d'amélioration par rapport au capteur traditionnel.

La tôle mistral de par sa géométrie qui se rapproche de celle du capteur à absorbeur étagé présente une bonne surface d'échange. Mais la minceur de l'absorbeur et la forte conductivité sont les éléments défavorables (un empilement de plusieurs tôles serait trop coûteux).
- Les éclats de verre sont intéressants parce qu'ils piègent l'infrarouge (ce que ne font pas ou peu les plastiques) et forment un lit relativement peu poreux $(p=0,5)$.

Il faut alors trouver une taille d'éclat optimum. Si les morceaux sont trop grands : l'échange est mauvais ; si ils sont trop petits : la réflection est importante. C'est ce que montre avec évidence la figure $3 a$.

Le verre sélectif horti + couramment utilisé permet des rendements comparables aux meilleures tôles et mousses plastiques car il a un coefficient d'émissivité faible.

- Enfin la mousse plastique Retipren présente une très bonne surface d'échange ( 10 à 20 fois plus importante que pour les éclats de verre) et c'est ce qui lui donne un si bon rendement malgré sa porosité très forte $(p=0,97)$.

5. Conclusion. - Nos recherches vont maintenant porter dans deux directions.

- Sur une mousse alvéolaire de porosité plus faible à condition que cela ne se fasse pas au détriment de la surface d'échange.

- Sur l'utilisation des propriétés sélectives du verre horti + avec une géométrie qui favorise les échanges et oriente les surfaces traitées vers le soleil.

Nous espérons aboutir à la définition d'un matériau poreux rustique adapté à la constitution de parois utilisables dans l'habitat pour le captage de l'énergie solaire par renouvellement d'air. Cet élément de paroi isolant à l'état naturel pourrait être associé à un stockage localisé à chaleur latente du type de ceux qui ont été étudiés dans le mur diode [18] (Jaffrin A., Bourdeau L.).

\section{Bibliographie}

[1] Schneider, M., Sylvain, J. D., Berger, X., Jaffrin, A. et BOURDEAU, L., Matériau absorbeur et accumulateur de calories sous forme de chaleur latente et applications. Brevet ANVAR-CNRS 79/13296.

[2] Meinel, A. B. and Meinel, M. P., Appl. sol. Energy (AddisonWesley Publishing Company) p. 383.

[3] Beckman, W. A. and Duffie, W. A., Solar Energy thermal processes (Wiley-Interscience publication) p. 165.

[4] Cadiergues, R., Colloque Avignon. CSTB (1978).

[5] Francia, G., Sol. Energy 12 (1968) 51.

[6] Buchberg, H., Lalude, D. A. and Edwards, D. K., Sol. Energy 13 (1971) 193.

[7] Buchberg, H., Lalude, O. A., Sol. Energy 13 (1971) 223 .

[8] SelcuK, K., Sol. Energy 13 (1971) 165.

[9] Chiou, J. P., Duffie, J. A. and EL WaKIL, M. N., Sol. Energy $9(1965) \mathrm{n}^{\circ} 2$.
[10] Pince, C., Thèse Université de Perpignan 1978.

[11] Grossin, R., Pellerin, J. F., Sté Bertin Document NN 5078-36.

[12] Beckman, W. A., J. Eng. power. Trans. ASME Series A 90 (1968) 51.

[13] Hamid, Y. H. and Beckman, W. A., J. Eng. POWER. ASME Paper no 69-WA/Sd-6 (1969).

[14] Hamid, Y. H. and Beckman, W. A., J. Eng. POWER (avril 1971) 221

[15] Document technique EDF lampes Sylvania code $n^{\circ} 1272$.

[16] Urbain, G., Manuel de l'Ingénieur R 2720.

[17] Comolet, R., Mécanique expérimentale des fluides (Masson).

[18] JAFFrin, A. et BourdeaU, L., Actual performance of a latent heat diode wall. Colloque d'Izmir (août 79). 